\title{
Correspondence
}

\section{Queering the Mental Health Model}

I have been involved in a project run by the artist La JohnJoseph (JJ) on 'Queering the Mental Health Model'. JJ's interest in the area was sparked by their experience visiting an ex-partner who was detained on a psychiatric ward, where 'queerness' often seemed to be conflated with illness by ward staff. JJ observed the difficulty of maintaining individuality in the confines of the ward environment; they noted that an attempt to do so was at times interpreted by staff as a sign of pathology.

JJ led 10 sessions over 12 months around the UK, to which people who self-identify as queer were invited to talk about their experience of psychiatric services. There was a general sense that people felt poorly understood outside specialist services, which are difficult to access; participants described a sense of having to fight their way through the system. There was, however, a multitude of positive feedback from people who had felt able to access a small number of appropriate specialist services.

'Queer' tends to be used now as an umbrella term to describe non-cis or non-heterosexual people. Historically derogatory, from the early 1990s, queer was reclaimed as a self-affirming term, originally by the gay rights movement. Queer challenges our tendency towards conventional categorisation into defined groups and helps remind us of individuality and the diversity of identity. As part of the LGBTQ community, it is known that people who self-identify as queer suffer higher rates of mental distress than the population at large. ${ }^{1} \mathrm{JJ}$ 's project will shed some light on the reasons for this.

There is a dearth of literature on this topic. A literature search by Reay House Library (based at Lambeth Hospital in London) on the 'experience of psychiatric/mental health services of people who self-identify as queer' found a total of 33 articles, of which four were by UK authors. None of these had a specific focus on people who identify as queer; they described experiences of the UK LGBTQ community in general. Two articles have an historical focus. Hughes et al have published two recent articles, one looking at the experiences of LGBTQ youth (ages 16-25) of suicidality and help-seeking ${ }^{2}$ and one on the perceptions and practice of mental health staff of this group. ${ }^{1}$ There is clearly scope to further examine the experience of psychiatric services of people who identify as queer, to help inform how these might better meet demand and reduce feelings of distress and marginalisation.

$J$ J's performance piece based on their experiences on the psychiatric ward, A Generous Lover, will tour the UK in September. There is further information at www.lajohnjoseph.com.

Andrew Camden, ST6 in General Adult Psychiatry, South London and the Maudsley NHS Foundation Trust, UK. email: Andrew.Camden@doctors.org.uk

1 McDermott E, Hughes E, Rawlings V. Mental health staff perceptions and practice regarding self-harm, suicidality and help-seeking in LGBTQ youth: findings from a cross-sectional survey in the UK. Issues Ment Health Nurs 2018; 39(1): 30-6

2 McDermott $E$, Hughes $E$, Rawlings $V$. Norms and normalization: understanding lesbian, gay, bisexual, transgender and queer youth, suicidality and help-seeking. Cult Health Sex 2018; 20(2): 156-72. doi:10.1192/bjb.2019.45

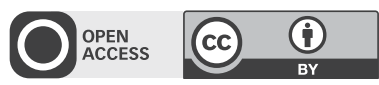

(c) The Author 2019. This is an Open Access article, distributed under the terms of the Creative Commons Attribution licence (http://creativecommons.org/ licenses/by/4.0/), which permits unrestricted re-use, distribution, and reproduction in any medium, provided the original work is properly cited.

\section{Effects of suicide on psychiatry trainees}

We read with interest the survey by Gibbons et al and welcomed the idea of examining what kind of support may help clinicians deal with this difficult aspect of mental health practice.

It is interesting to note how some responses to the survey indicated that having experienced a patient suicide as a trainee had a significant influence on the responder's choice of subspecialty. This finding highlights how junior doctors, whether in a formal training programme or not, are particularly vulnerable to the adverse effects of a patient suicide, with potential effects on recruitment and retainment in psychiatry.

In our mental health trust in South East London, trainee-led initiatives in collaboration with the postgraduate training department have been making changes to the learning and support offered to junior doctors who are involved in a serious incident investigation in the past 5 years. ${ }^{2}$ The projects involve annual events to promote knowledge and discussion about quality and safety; discussions about the process of serious incident investigations and the support available at each junior doctor induction; and the development of a written resource on formal and informal sources of support for junior doctors involved in a serious incident investigation. These projects are under constant review in order to respond to trainee feedback.

We thank the authors for the valuable work exploring how suicide can affect doctors of any grade. We hope that mental health trusts and postgraduate training departments continue working to develop formal and informal support structures for doctors experiencing this difficult event.

Marilia Calcia, Consultant Liaison Psychiatrist, South London and Maudsley NHS Foundation Trust, UK. email: marilia.calcia@nhs.net; Alice Debelle Specialist Registrar in Child and Adolescent Psychiatry, South London and Maudsley NHS Foundation Trust, UK

1 Gibbons R, Brand F, Carbonnier A, Croft A. Effects of patient suicide on psychiatrists: survey of experiences and support required. BJPsych Bull 2019; doi: 10.1192/bjb.2019.26.

2 Calcia M. Why adverse incidents are an opportunity for learning. $B M$ 2016; 355: i5069.

doi:10.1192/bjb.2019.46

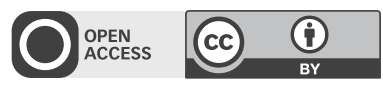

(c) The Authors 2019. This is an Open Access article, distributed under the terms of the Creative Commons Attribution licence (http://creativecommons.org/ licenses/by/4.0/), which permits unrestricted re-use, distribution, and reproduction in any medium, provided the original work is properly cited. 